\title{
Numerical Simulations of the Rayleigh-Taylor Instability
}

\author{
Grétar Tryggvasun \\ Department of Mechanical Engineering and Applied Mechanics, \\ The Unitersity of Michigan, Ann Arbor, Mishigan 48100 \\ Received February 7, 1986; revised May 15, 1987
}

\begin{abstract}
Two Lagrangian-Eulerian vortex methods to simulate the motion of an interface between inviscid fluids of different densities are presented. The representation of the interface as a vortex sheet eliminates numerical diffusion, and by coupling the tracked interface with a stationary grid (using the well-known vortex-in-cell method) the high cost associated with traditional vortex methods is reduced. These methods are applied to the Rayleigh-Taylor instability. For finite density ratios the appearance of rolled up vortices and a possibie singularity formation has limited simulations in the past. By providing proper regularization our methods overcome some of these difficulties. 1988 Academic Press, Inc.
\end{abstract}

\section{INTRODUCTION}

One of the classic examples of hydrodynamic instability is the mixing of wo fluids that takes place if a heavy fluid initially lies above a lighter one in a gravitational field. The first to investigate this problem was Lord Rayleigh [43], who late in the last century considered the linear stability problem for inviscid fluids with various stratification profiles, including sharply stratified fluids. In the early fifties G. I. Taylor [51] renewed interest in this problem by pointing out that it is formally identical to the problem of an interface accelerated toward the heavy fluid. This observation made controlled laboratory experiments possible, and at Taylor's suggestion D. J. Lewis [29] performed several such experiments, confirming Taylor's and Rayleigh's theoretical predictions for the linear stage and shedding some light on the large amplitude evolution. Several investigators have since repeated Lewis' experiments using devices ranging from rubber bands (Emmons, Chang, and Watson [18]) to rocket motors (Read [42]) to produce the desired acceleration.

The stability analysis of Rayleigh and Taylor has been extended to take into account other physical effects such as surface tension and viscosity (Bellman and Pennington [8], Chandrasekhar [13]), compressibility (Mitchner and Landshoff [36]), and weakly nonlinear amplitudes (e.g., Emmons et al. [18]). Although these analytical investigations have revealed scveral interesting properties, most have not addressed the large amplitude behavior of a single bubble, or the interactions of these bubbles at large amplitudes. For a single bubble of negligible density penetrating the heavy fluid the asymptotic shape at large amplitudes has been 
analyzed by Birkhoff and Carter [11] and Garabedian [19]. For discussions of recent results see Garabedian [20]. Experimentally, the large amplitude regime is (relatively) easily reached and is found to consist of many competing and interacting waves of different amplitudes and wavelengths. The dominant length scale at large amplitudes is often considerably larger than at small (linear) amplitudes, and for large density differences the final stage in experiments often consists of a single bubble, whose size is determined by the experimental apparatus. This increase in the mean length scale is well known from some other nonlinear systems such as shear layers (Aref and Siggia [3]) and interfaces in a Hele-Shaw cell (Tryggvason and Aref $[55,56]$ ) and is, for the most part, a mechanism outside the domain of analytical investigation. The shortcomings of the above-mentioned analytical investigations are shared by the various models that have been advanced for the Rayleigh-Taylor instability. Most consider only the growth of a single wavelength initial perturbation (e.g., the celebrated Fermi model mentioned by Birkhoff [10]), and those that address the large amplitude mixing are usually not founded on sufficiently strong physical arguments to be expected to give trustworthy results a priori. See, for example, the model of Sharp and Wheeler and Sharp's [50] discussion of the model. Most such models deal with the case where the lighter fluid has negligible density. For a model of the small density case see Aref and Tryggvason [4].

Considering the difficulties in the analytical treatment of the Rayleigh-Taylor instability, it is not surprising that it has been the subject of many numerical studies. The earliest numerical simulations are probably those described by Birkhoff [10], but the first successful studies are attributed to Harlow and Welch [22] and Daly [16] who used the marker-and-cell method. Daly studied the evolution of a single wavelength initial (velocity) disturbance for various density ratios and established the now well-known behavior of a single wave at large amplitudes. For small density differences the interface rolls up into two counter-rotating vortices. For larger density differences these vortices are smaller and move toward the light fluid. For sufficiently large density differences no roll-up is observed and the heavy fluid falls in smooth, pointed spikes into the light fluid, while the light fluid forms big rounded bubbles that propagate into the heavy fluid. Daly's "classic" computer generated pictures are reproduced by Lugt [31]. Later numerical studies have tried to achieve higher accuracy and larger amplitudes. Although considcrablc progress has been made, generally these studies have only reached amplitudes of once or twice the perturbation wavelength and have been confined to relatively simple initial conditions. Only for one case, an inviscid fluid falling into a vacuum (sometimes referred to as the single fluid case), has an accurate solution been obtained. The results were obtained independently by Baker, Meiron, and Orszag [6] using a vortex method and by Menikoff and Zemach [35] using a conformal mapping technique. Although Baker et al. where able to follow the evolution of an interface pcrturbed by a single wave for as long as they wished, more general initial conditions (many waves) led to problems that ultimately brought the computations to a halt (Orszag; private communication, 1984). For the two fluid case when vor- 
tices form on the side of the spike, most methods appear to fail on the roll-up. Baker et al. report only limited success, and other methods based on tracking the interface appear to do no better. For recent studies see, e.g., Glimm, McBryan. Menikoff, and Sharp [21]; Pullin [40]; and Youngs [59]. Some success has been achieved by using diffusive codes that do not maintain the sharp interface at all times (see, e.g., Youngs [59]), but the accuracy obtained and the relevance to the sharply stratified model most commonly studied is not clear.

Although the Rayleigh-Taylor instability is rarely observed in its "purest" form, it plays an important role in various natural and technological processes. Often the motion is modificd by additional processes such as phase changes, and the initial setup is frequently such that "instability" is a somewhat misleading description. Any motion of one fluid in another of different density is, of course, governed by essentially the same mechanism as the one governing the nonlinear Rayleigh-Taylor instability even if there is no stable state initially. The formation of bubbles from a vapor film beneath a liquid in film boiling is a classic example of relatively "pure" Rayleigh-Taylor instability. In technological fields such diverse applications as the formation of droplets by the vibration of a liquid with a free surface to the break-up of accelerated thin-shell laser fusion targets $[17,57]$ may be mentioned. Severa? geological and physical phenomena are also believed to be essentially due to Rayleigh-Taylor instability [41].

In addition to its intrinsic importance the Reyleigh-Taylor problem has become a popular test case for numerical methods intended to study multiphase or multimaterial problems $[21,24,58]$. For further discussion of the Rayleigh-Taylot instability the reader is referred to the review by Sharp [50].

In this paper the evolution of the Rayleigh-Taylor instability will be studied by approximating the fluids as inviscid, incompressible, and each of constant density. The interfacial surface tension is taken to vanish. Although this model is possibiy the simplest one capable of capturing the physics involved, and hence the one most commonly studied, it was already discovered by Birkhoff [10] that the complete neglect of stabilizing mechanisms, such as viscosity or surface tension or both, leads to certain difficulties. An indication of these difficulties is already evident in the linearized stability analysis, which gives the growth rate of an infinitely small unstable wave as being proportional to the square root of its wavenumber. This nonphysical behavior (that infinitely short waves grow infinitely fast) led Birkhoff: to speculate that the initial-value problem might be ill-posed, and that an initial analytic interface might not remain analytic at all times. Ill-posedness and unboun ded growth rates may be only a necessary condition for singularity formation which must be due to nonlinear interactions that excite smaller and smaller length scales. Unbounded growth rates can, however, lead to numerical problems, even in the linear stage, since a small error can amplify rapidly. (Nonlinear interactions can also stabilize small waves, for example, the single fluid Rayleigh-Taylor instability, which does not form rolled-up vortices, is believed not to form a singularity [6].)

For regular vortex sheets (with no density difference) there exist fairly strong numerical and analytical evidence (Meiron, Baker, and Orszag [33]: Moore [38]; 
Krasny [26]; Higdon and Pozrikidis [23]) that after a finite time the vortex sheet forms a curvature singularity that becomes the center of vigorous roll-up. The singularity seems to be due to the strong advection of vorticity into the point where the singularity forms. Just before the singularity forms, the vortex sheet strength has a sharp spike at that point. (This is true only for the initial motion. At large amplitudes no concentration of the vorticity is believed to take place, see Saffman and Baker [46]). When the vortex sheet is modelled as a row of point vortices, the model generally resists efforts to reliably follow the roll-up numerically beyond the time singularity forms. However, it is well known that the physical system being modelled by a vortex sheet (and which differ from the model by having some viscous effects and finite thickness) can typically exhibit roll-up, and the rolled up region is quite regular long after the numerical simulations have broken down.

It seems to be the general belief that even though the vortex sheet model breaks down after a finite time, a "weak" solution still exists, relevant to the physical system modelled, which may be obtained by a proper regularization. The situation may be somewhat analogous to shocks in compressible, inviscid fluids, which are captured by the proper artificial viscosity. Various regularizations have been tried. A common one is to replace the innermost part of the vortex by a singlc point vortex and to move all vortices that come within a certain distance of this center vortex into it (see, e.g., Moore [37]). Another approach is to replace the singular point vortices by smooth vortex blobs (see, e.g., Chorin and Bernard [14]). In the case of stratified flow, where it is necessary to maintain a continuous interface, it appears that this second method has certain advantages. Recently, this desingularization has been used to study the behavior of the inner rolled-up spiral on a vortex sheet. Krasny [27] has studied the large amplitude Kelvin-Helmholtz instability, and Anderson [1] has studied the late time deformation of a rising cylinder of slightly buoyant fluid (where the Boussinesq approximation is applicable). Our interest in this second regularization is due to the observation that the vortex-in-cell method, which is used in this study, has properties very similar to the vortex blob methods (see Tryggvason [54]). For additional discussions of regularization in interface simulations, see $[60]$.

The rest of the paper is laid out as follows. In Section 2 the governing equations are presented, and two grid based vortex methods for simulations of sharply stratified flow are presented. Computational examples are presented in Section 3. The methods are compared, and the reason why our methods successfully deal with the difficulties that limited simulations in the past are discussed. Section 4 contains concluding comments and examples of more demanding simulations.

Short accounts of the methods and preliminary results were presented at the American Physical Society, Division of Fluid Dynamics annual meeting in Providence, RI., Nov. 18-20, 1984, and in Tucson, AZ, Nov. 22-26, 1985, and at the SIAM spring meeting in Pittsburgh, PA, June 24-26, 1985. Some of this material is available in the thesis by Tryggvason [53]. 


\section{Governing Equations and Numerical Method}

If the interface between two inviscid fluids of different densities is not perpendicular to the pressure gradient, vorticity will be generated as prescribed by Bjerknes' generalization of Kelvin's circulation theorem. In the interior of each fluid the original theorem of Kelvin holds. Hence, the vorticity must be confined to the interface at all times and can therefore be regarded as a generalized vortex sheet. The formulation of the problem in terms of a vortex sheet greatly reduces the computational effort, since now it is necessary only to follow the motion and strength of the shcet itself. No interior degrees of freedom need to be considered. Given the position and strength of the vortex sheet, its velocity is given by Birkhoff's integral formula $[10]$,

$$
\mathbf{U}(s, t)=\frac{1}{2 \pi} P \int \frac{\hat{k} \times\left(\mathbf{x}(s, t)-\mathbf{x}\left(s^{\prime}, t\right)\right)}{\left|\mathbf{x}(s, t)-\mathbf{x}\left(s^{\prime}, t\right)\right|^{2}} \gamma^{\prime}\left(s^{\prime}, t\right) d s^{\prime},
$$

where the principal-value integral is taken along the sheet. Here $\mathbf{U}$ is the velocity of the vortex sheet, $\mathbf{x}$ its position, $\gamma$ the vortex sheet strength, and $s$ an archlength coordinate. Once the velocity is found, a new position can be determined by integrating

$$
d \mathbf{x} / d t-\mathbf{U} .
$$

Usually the vortex sheet is modelled by a row of point vortices and Birkhoffs integral is approximated by a summation. Since it is necessary to sum over all the vortices in order to find the velocity of each vortex, $O\left(N^{2}\right)$ operations are reeded per time step, where $N$ is the number of vortices. Obviously for large $N$ finding the velocities becomes quite demanding on computational resources. In the present study a different approach is taken. Instead of using Birkhoff's integral (1) to find the velocities of the (singular) vortex sheet, we use the fact that a stream function $\psi$ corresponding to the velocity field $\mathbf{u}(x, y)$ induced by the vortex sheet satisfies

$$
\nabla^{2} \psi=-\omega ; \quad \mathbf{u}-\nabla \times \psi \hat{k} .
$$

where

$$
\omega(\mathbf{x})=\int \delta(\mathbf{x}(s, t)-\mathbf{x}) \gamma(s) d s
$$

is the vorticity and here $\delta(\mathbf{x})$ is a two-dimensional delta function. When the sheet is approximated by a row of point vortices, $i=1, \ldots, N$ of circulation $\Gamma_{i}$, the vorticity is approximated as

$$
\omega(\mathbf{x})=\sum \delta\left(\mathbf{x}_{i}(t)-\mathbf{x}\right) \Gamma_{i},
$$

where

$$
\Gamma_{i}=\int_{1, i} \gamma(s) d s
$$


and $\Delta s_{i}$ is the length of the sheet modelled by vortex $i$, and $\mathbf{x}_{i}(t)$ is its position. The benefits of using (3) directly, instead of its formal solution (1), arise from the fact that for a regular domain with simple boundary conditions the Poisson equation (3) can be solved quite efficiently. Fast Poisson equation solvers require $O\left(M^{2} \log M\right)$ operations for the inversion on a $M \times M$ grid, and for highly contorted interfaces we may assume approximately one vortex per grid square, so the operations needed to find the velocities are $O(N \log N)$, considerably fewer than the $O\left(N^{2}\right)$ required by a direct summation method. Fast Poisson solvers generally require fairly simple boundary conditions. In the calculations reported here the computational domain is a rectangular box with periodic boundaries in the horizontal $(x)$ direction and rigid top and bottom.

Since the source term in Eq. (3) is a (singular) line source whose position will not (in general) coincide with the grid lines of a regular grid, some preprocessing is required before a standard Poisson equation solver can be invoked. One way of doing this is to generate an irregular finite element grid such that the interface (the line source) always follows element boundaries. Another method is to generate a curvilinear, finite difference grid such that the interface coincides with a grid line. Both of these methods requirc somewhat expensive grid construction. in addition to the more serious consequence that the resulting system of equations is not as efficiently solvable as when a regular grid is used. In our approach, we keep a fixed regular grid and use a vortex-in-cell (VIC) algorithm similar to the one described more than a decade ago by Christiansen [15]. In the original VIC method each point vortex making up the vortex sheet interface is split into four pieces. and each piece is assigned to the nearest grid point by the so-called area-weighting rule. When all the point vortices have been assigned to grid points, the singular vortex sheet has been replaced by a smoother vorticity distribution that is used by the Poisson solver. Although at each time step a smooth version of the vorticity is used to find the velocities, the vortex sheet interface is always well defined and does not diffuse. The original method, smoothing the vorticity only to the nearest four grid points, produces some anisotropy on the smallest scales. This is a well-understood problem and can be corrected (in those cases where such small-scale anisotropy may cause difficulties) by creating a smoother, more isotropic grid vorticity from the singular point vortex field. For early work on smooth, or "quiet," VIC methods see, e.g., Hockney, Goel, and Eastwood [25]; Buneman [12]; and Leonard [28]. For recent work see the paper by Anderson [2], which extends ideas presented by Mayo [32]. In this paper we have used both the original four-point area-weigthing rule and a smoother interpolation function suggested by Peskin [39] in a slightly different context. Since the procedure is well described by Peskin [39], and a short discussion is given by Tryggvason [54], we will not describe it further here. For a description of the original method the reader is referred to Christiansen's paper [15], Baker [5], Aref and Siggia [3], and Leonard [28].

In the original VIC method the circulation of each point vortex is assumed to remain constant, since the method was designed to simulate the motion of an inviscid, constant-density fluid. When the point vortices are used to represent a 
generalized vortex sheet interface separating different fluids, the vorticity of each material segment changes with time and an auxiliary equation for the evolution of the vortex sheet strength must be provided. The extension of the VIC method ic handle these cases was first considered by Meng and Thomson [34] for inviscid fluids and for flow in a Hele-Shaw cell. However, they only considered the case of weak stratification and presented a rather limited set of examples of applications. Tryggvason and Aref $[55,56]$ extended the method to arbitrary stratification for flow of two fluids in a Hele-Shaw cell. Tryggvason [52] used the version for weakly stratified inviscid flow to study the initial motion of gravity currents. The extensions to arbitrary stratification are discussed below.

Consider an interface between inviscid, incompressible, irrational fluids. The flow of each fluid is governed by Euler's equation

$$
\rho_{i} \mathbf{a}_{i}=-\nabla p_{i}-\rho_{i} g \hat{\jmath}, \quad i=1,2 .
$$

Here

$$
\mathbf{a}_{i}=\frac{\partial \mathbf{u}_{i}}{\partial t}+\mathbf{u}_{i} \cdot \nabla \mathbf{u}_{i}
$$

is the acceleration of a material point, $\rho_{i}$ is the density of fluid $i, p_{i}$ is the pressure in fluid $i, g$ is the gravitational acceleration, and $\hat{j}$ is a unit vector directed upward. We will adopt the convention that 1 (2) refers to the fluid initially at the bottom (top). See Fig. 1. To obtain an equation for the vortex sheet strength

$$
\gamma=\left(\mathbf{u}_{1}-\mathbf{u}_{2}\right) \cdot \hat{s}
$$

we subtract the tangential component of (7) across the interface to get

$$
\frac{1}{2}\left(\left(\rho_{1}+\rho_{2}\right)\left(\mathbf{a}_{1}-\mathbf{a}_{2}\right)+\left(\rho_{1}-\rho_{2}\right)\left(\mathbf{a}_{1}+\mathbf{a}_{2}\right)\right) \cdot \hat{s}--\frac{\partial}{\partial s}\left(p_{1}-p_{2}\right)-\left(\rho_{1}-\rho_{2}\right) g \hat{\jmath}
$$

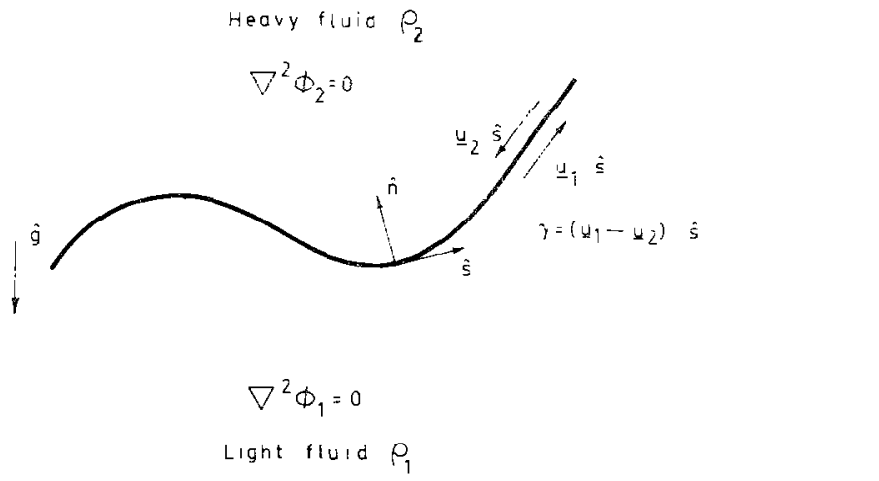

Fig. 1. Notation. The heavy fluid (2) is initially above the light fluid 11 ) Gravity acts downward. 
The velocity of the interface is defined to be the average of the velocities on either side:

$$
\mathbf{U}=\frac{1}{2}\left(\mathbf{u}_{1}+\mathbf{u}_{2}\right) .
$$

Using the definition for $\gamma$ and $\mathbf{U}$ we have

$$
\mathbf{u}_{1}=\mathbf{U}+\frac{1}{2} \gamma \hat{\boldsymbol{s}} ; \quad \mathbf{u}_{2}=\mathbf{U}-\frac{1}{2} \gamma \hat{\boldsymbol{s}} .
$$

Substituting this into the definition for $\mathbf{a}_{i}$, the convective acceleration, we obtain

$$
\mathbf{a}_{i}=\frac{d \mathbf{u}_{i}}{d t} \pm \frac{1}{2} \gamma \frac{\partial \mathbf{u}_{i}}{\partial s}
$$

where the $+(-)$ sign is for $i=1(2)$, and

$$
\frac{d \mathbf{u}_{i}}{d t}=\frac{\partial \mathbf{u}_{i}}{\partial t}+\mathbf{U} \cdot \nabla \mathbf{u}_{i}
$$

is the rate of change in $\mathbf{u}_{i}$ following an interface point. Substituting the above results into (10) we obtain the equation governing the evolution of the vortex sheet strength $\gamma$ :

$$
\frac{d \gamma}{d t}+\gamma \frac{\partial \mathbf{U}}{\partial s} \cdot \hat{s}=2 A \mathbf{a} \cdot \hat{s}+2 A g \hat{J} \cdot \hat{s}-\frac{2}{\rho_{1}+\rho_{2}} \frac{\partial\left(p_{1}-p_{2}\right)}{\partial s} .
$$

Here $A$ is the Atwood ratio

$$
A=\frac{\rho_{2}-\rho_{1}}{\rho_{2}+\rho_{1}}
$$

and

$$
\mathbf{a}=\frac{1}{2}\left(\mathbf{a}_{1}+\mathbf{a}_{2}\right)
$$

is the average of the acceleration above and below the interface. Notice the difference between a and the acceleration of an interface point $d \mathbf{U} / d t$. The relation between these two (the tangential components) is easily shown to be

$$
\mathbf{a} \cdot \hat{s}=\frac{d \mathbf{U}}{d t} \cdot \hat{s}+\frac{1}{8} \frac{\partial \gamma^{2}}{\partial s}
$$

The difference between the pressures on either side vanishes for negligible surface tension or can be expressed by Laplace's equation $p_{1}-p_{2}=\sigma(1 / R)$, where $\sigma$ is the surface tension coefficient and $R$ is the radius of curvature. Equation (15) can therefore be written in terms of interface quantities only. 
Equation (15) and various other slightly different forms have been derived and rederived by many investigators. See, for example, Zaroodny and Greenberg [62]; Zalosh [61] (and the correction by Rottman and Olfe [45]); Baker, Meiron, and Orszag $[6,7]$; and Glimm et al $[21]{ }^{1}$

In the actual computation it is convenient to work in nondimensional variables, so we now proceed to derive the nondimensional form of the equation governing the vortex sheet strength generation, Eq. (15). The nondimensional form also allows us to make an important observation about small stratification. The width (W) of the computational box will be taken as the length scale. For interfaces perturbed by a single wave, or that contain a length scalc sct by a nonzcro surface tension, the wavelength of the single wave or the surface tension length may be the more relevant length. However, we choose $W$ for two reasons. For interfaces perturbed by many different waves, $W$ may be the only uniquely defined length scale, and second, it is convenient to work with a computational box of unit width. For the simulation of one wave the wavelength and the box width usually coincide. A time scale is now given by $\sqrt{W / A g}$ and the nondimensional variables (denoted by a tilde) are then defined as

$$
\mathbf{x}=W \tilde{\mathbf{x}}, \quad t=\sqrt{W / A g} \tilde{t} \quad(\mathbf{U}, \gamma)=\sqrt{\operatorname{AgW}}(\tilde{\mathbf{U}}, \tilde{\gamma}) .
$$

Substituting these into Eq. (15), dividing by a common constant, and dropping the tilde we get

$$
\frac{d \gamma}{d t}+\gamma \hat{s} \cdot \frac{\partial \mathbf{U}}{\partial s}=2 A \mathbf{a} \cdot \hat{s}+2 \frac{\partial y}{\partial s}-2 B \frac{\partial}{\partial s}\left(\frac{1}{R}\right)
$$

where

$$
B=\frac{\sigma}{A g\left(\rho_{1}+\rho_{2}\right) W^{2}}
$$

is a dimensionless surface tension coefficient (actually an inverse "Bond number"), and we have written $\hat{j} \cdot \hat{s}=\partial y / \partial s$. In this form it is easily seen that the terms representing the average acceleration become less and less important as $A \rightarrow 0$. For very small density differences we can neglect the term multiplied by $A$. This corresponds to making the Boussinesq approximation where the density is considered constant except when multiplying $g$. (Formally, this is the limit $g \rightarrow \infty$, $A \rightarrow 0$, but $A g$ is finite.) It is actually a trivial exercise to derive this limiting case by first making the Boussinesq approximation (see, i.e., Tryggvason [52]). The simplification achieved by the Boussinesq approximation is considerable, and in this limit the two numerical methods described below become identical.

I am grateful to Professor C. Dalton at the University of Houston for pointing out some of these references to me. 
Since the interface is parameterized by material points in the simulations, it is convenient to write the derivatives in (20) in terms of a material marker $\alpha$ rather than arclength $s$. Now

$$
\left.\left.\frac{\partial}{\partial t}\left(\gamma \frac{\partial s}{\partial \alpha}\right)\right)_{\alpha}=\frac{d}{d t} \gamma(s, t) \frac{\partial s}{\partial \alpha}+\gamma \frac{\partial}{\partial t}\left(\frac{\partial s}{\partial \alpha}\right)\right)_{x}
$$

since

$$
\left.\frac{\partial \gamma}{\partial t}\right)_{\alpha}=\frac{d}{d t} \gamma(s, t)
$$

The last term in $(22)$ is

$$
\gamma \hat{s} \cdot \frac{\partial \mathbf{U}}{\partial s} \frac{\partial s}{\partial \alpha}
$$

as is easily found by using the definition of arclength. Hence (20) can be rewritten as

$$
\frac{\partial}{\partial t}\left(\gamma \frac{\partial s}{\partial \alpha}\right)=2 A\left(\frac{\partial s}{\partial \alpha}\right) \mathbf{a} \cdot \hat{s}+2 \frac{\partial y}{\partial \alpha}-2 B \frac{\partial}{\partial \alpha}\left(\frac{1}{R}\right)
$$

When the interface is modeled as a row of point vortices, each point carries the circulation $\Gamma_{i}$ equal to the vortex sheet strength integrated over a small material line around the point. The circulation of a material line between points denoted by $\alpha^{-}$and $\alpha^{+}$is

$$
\Gamma=\int_{s\left(x^{-}\right)}^{s\left(\alpha^{+}\right)} \gamma(s) d s
$$

where $s$ is the arclength. Changing variables from $s$ to $\alpha$ we have

$$
\Gamma=\int_{\alpha^{-}}^{\alpha^{+}} \gamma(\alpha, t) \frac{\partial s}{\partial \alpha} d \alpha
$$

The time derivative of $\Gamma$ is

$$
\frac{d \Gamma}{d t}=\frac{d}{d t} \int_{\alpha^{-}}^{x^{+}} \gamma(\alpha, t) \frac{\partial s}{\partial \alpha} d \alpha=\int_{\alpha^{-}}^{\alpha^{+}} \frac{\partial}{\partial t}\left(\gamma \frac{\partial s}{\partial \alpha}\right) d \alpha,
$$

since $\alpha^{+}$and $\alpha^{-}$do not depend on time. Here the partial differentiation means that $\alpha$ is kept constant.

Equation (23), or (26), is the evolution cquation for the circulation of each point vortex needed to supplement Eqs. (2) and (3). However, the system of equations is not closed yet, since $\mathbf{a} \cdot \hat{s}$ has to be found. The most straightforward way to do so is 
to use the fact that the pressure is a potential for $\rho \mathbf{a}$ and an equation for $p$ is easily found by taking the divergence of Euler's equation. This is, for example, the method used by Glimm et al. [21]. For our purposes this has some drawbacks. The first one is that the pressure equation is a nonseparable elliptic equation, whereas we would prefer a simple Poisson equation so that a fast Poisson solver may be used. The second drawback is that since the pressure is a potential for $\rho$ a it is necessary to know on which side of the interface we are to find a. Since our intention is to avoid any complicated and expensive grid constructions, it is essential not to have to make distinctions between the two fluids, except for the iocation of the interface. Fortunately, both of these difficulties can be overcome in a relatively simple way, although at the cost of having to solve several Poisson's equations in an iterative procedure. Two methods have been developed to treat this term, both of which depend on some iterative solution of the Poisson equation. The distinction between the two methods is dependent on Eq. (18). One method uses the decomposition on the right-hand side of (18), whereas the other method works with a somewhat different decomposition of a. We describe this second method, which we refer to as the acceleration potential (AP) method, first.

Using the fact that any vector field can be written as the sum of a divergence-free part and a curl-free field, we introduce two potentials, $\theta$ and $\phi$ (see, e.g., Serrin [47]) such that

$$
\mathbf{a}=\frac{\partial \mathbf{u}}{\partial t}+(\mathbf{u} \cdot \nabla) \mathbf{u}=\nabla \theta-\nabla \times(\phi \hat{k})
$$

Now a denotes the acceleration field in the whole computational domain, and not only on the interface. For fluid of constant density, $p_{0}$, it is of course obvious that $\phi=$ constant. and

$$
\theta=-\frac{p}{\rho_{0}}-g z
$$

To find equations for $\theta$ and $\phi$ the definition of a is used. Taking the divergence of Eq. (27) we obtain

$$
\begin{aligned}
\nabla^{2} \theta & =\nabla \cdot\left(\frac{\partial \mathbf{u}}{\partial t}+(\mathbf{u} \cdot \nabla) \mathbf{u}\right)=\nabla \cdot(\mathbf{u} \cdot \nabla) \mathbf{u}=2 \frac{\partial v}{\partial x} \frac{\partial u}{\partial y}-2 \frac{\partial u}{\partial x} \frac{\partial v}{\partial y} \\
& =-2\left(\frac{\partial^{2} \psi}{\partial x^{2}} \frac{\partial^{2} \psi}{\partial y^{2}}-\left(\frac{\partial^{2} \psi}{\partial x \partial y}\right)^{2}\right),
\end{aligned}
$$

where $\mathbf{u}=(u, v)$ and we have used the fact that $\nabla \cdot u=0$. Taking the curl of Eq. 27$)$ yields

$$
\nabla^{2} \hat{\phi} \hat{k}=\nabla \times \frac{\partial \mathbf{u}}{\partial t}+\nabla \times(\mathbf{u} \cdot \nabla) \mathbf{u}=\frac{d \omega}{d t} \hat{k}=\sum_{i} \delta\left(\mathbf{x}_{i}-\mathbf{x}\right) \frac{d \Gamma_{i}}{d i} \hat{k}
$$


Although, in general, a is discontinuous across the interface, we treat it in the same way as the velocities, that is, we smooth the source terms for its potentials onto the grid and hence obtain a smooth approximation for a. The equation for $\phi$ has exactly the same form as the equation for the stream function $\psi$, therefore it is possible to use the VIC program directly. (The boundary conditions are also the same as will be shown shortly.) Since the source terms (Eq. 15) contain the acceleration a, an iterative procedure is necessary. The technique to solve nonseparable elliptic equations by iterative application of a fast Poisson solver, described above, has been discussed in more detail by Bernhardt and Brackbill [9].

The source term for the $\theta$ equation is easily shown to consist of a part that is nonzero within each fluid but discontinuous across the interface, and a line source at the interface (Tryggvason [53], Glimm et al. [21]). The line source is a consequence of the discontinuous velocity field and, since the replacement of the singular vortex sheet by a smooth vorticity field gives a smooth and continuous velocity field, the line source should not be added explicitly in our treatment. The source term can therefore be obtained in a way consistent with previous approximations by numerical differentiation of the velocity field in the whole computational domain. This obviously leads to considerable simplification in implementation and allows the computations to go much faster. The differentiation, though, has to be done somewhat carefully. As shown below, $\theta$ has Neumann boundary conditions (equal to zero) at the rigid boundaries. The integral of the source term over the domain must therefore vanish, and it is important that the discretization preserves this property. A straightforward discretization of (29) using standard three-point central differences for the stream function does not. However, using central differences on the velocity field does have this conservation property, but since the velocity is found by central differences from the stream function, the result is a scheme that involves the stream function from five grid points in each direction. It is possible to use the three-point formula and enforce conservation by adding or subtracting a small correction from the value at each grid point. For a discussion of the problem, and this procedure, see Roache [44]. Although this more compact formula is preferable, since it corresponds to smaller blobs, it was found to be susceptible to instabilities at large amplitudes, particularly when the original VIC method was used. We have therefore used the following conservative differencing in the runs presented here:

$$
\begin{aligned}
\left(\frac{\partial^{2} \psi}{\partial x^{2}} \frac{\partial^{2} \psi}{\partial y^{2}}-\left(\frac{\partial^{2} \psi}{\partial x \partial y}\right)^{2}\right)= & \left(\left(\frac{\psi_{i+2, j}+\psi_{i-2, j}-2 \psi_{i, j}}{4 \Delta x^{2}}\right)\left(\frac{\psi_{i, j+2}+\psi_{i, j-2}-2 \psi_{i, j}}{4 \Delta y^{2}}\right)\right. \\
& \left.-\left(\frac{\psi_{i+1, j+1}-\psi_{i+1, j-1}-\psi_{i-1, j+1}+\psi_{i-1, j-1}}{4 \Delta y \Delta x}\right)^{2}\right) .
\end{aligned}
$$

We now derive the relevant boundary conditions for $\theta$ and $\phi$. The computational domain is a box with a rigid top and bottom, $y=0, L$, and is periodic in the 
$x$-direction. Thus, boundary conditions are that the normal velocity, $v$, vanishes at the top and bottom boundaries. The normal acceleration

$$
a_{y^{\prime}}=\frac{\partial v}{\partial t}+u \frac{\partial v}{\partial x}+v \frac{\partial v}{\partial y}
$$

must therefore also vanish. For the acceleration potentials $\theta$ and $\phi$ this means

$$
\frac{\partial \theta}{\partial y}+\frac{\partial \phi}{\partial x}=0 . \quad \text { on } y=0, L .
$$

The decomposition formula for the acceleration leaves a certain freedom in the choice of $\theta$ and $\phi$. We want to show that without a loss of generality we can take $\partial \theta / C y=0$ and $\phi=0$ as boundary conditions on $y=0, L$. From the definition of $\theta$ and $\phi(27)$, it is obvious that we can add $\theta^{\prime}$ to $\theta$ and $\phi^{\prime}$ to $\phi$ without affecting the physical accelerations if $\theta^{\prime}$ and $\phi^{\prime}$ satisfy

$$
\nabla \theta^{\prime}=\nabla \times\left(\phi^{\prime} \hat{k}\right)
$$

or

$$
\frac{\partial \theta^{\prime}}{\partial x}=\frac{\partial \phi^{\prime}}{\partial y} ; \quad \frac{\partial \phi^{\prime}}{\partial x}=-\frac{\partial \theta^{\prime}}{\partial y}
$$

That is, $\theta^{\prime}$ and $\phi^{\prime}$ must satisfy the Cauchy-Riemann equations. Assume now shat $\partial \theta / \partial y \neq 0$ and $\phi \neq 0$ on $y=0, L$. Then we can find $\theta^{\prime}$ and $\phi^{\prime}$ satisfying

$$
\begin{array}{ll}
\nabla^{2} \theta^{\prime}=0 ; & \theta^{\prime}=-\frac{\partial \theta}{\partial !} \quad \text { on } \quad y=0, L, \\
\nabla^{2} \phi^{\prime}=0 ; & \phi^{\prime}=-\phi \quad \text { on } \quad y=0, L .
\end{array}
$$

Making the gauge transformation

$$
\theta^{\prime \prime}=\theta+\theta^{\prime} ; \quad \phi^{\prime \prime}=\phi+\phi^{\prime},
$$

we obtain potentials $\left(\theta^{\prime \prime}, \phi^{\prime \prime}\right)$ which give the same physical acceleration as $\left(\theta_{,} \phi\right)$ and satisfy the stated boundary conditions.

In the second method (referred to as the iterative time-step (ITS) method) we still use the VIC method to find the velocities efficiently from the vorticity, but instead of using the smoothed vorticity (and velocity) field to calculate the grid acceleration for (15), we work with the decomposition of the mean acceleration inte the acceleration of an interface point and a convective term involving only the vortex sheet strengths. Eq. (18). The term containing $\gamma$ is easily found at each time step, but $d \mathbf{U} / d t$ is found as follows. First the interface is advanced and the vortex 
sheet strength updated using $d \mathbf{U} / d t$ from the last time step as an approximation. Then the velocities at the new position are found, using the new approximate value of $\gamma$ (or $\Gamma$ ). These velocities are then used to calculate $d \mathbf{U} / d t$ (by finite differencing) for the last position and the time-step is repeated. This iteration is repeated until satisfactory convergence is achieved. Since it is somewhat difficult to implement this second method using the time-stepping package for the first method (STEP of Shampine and Gordon [491), a second-order predictor-corrector was used for this method.

The Acceleration Potential Method

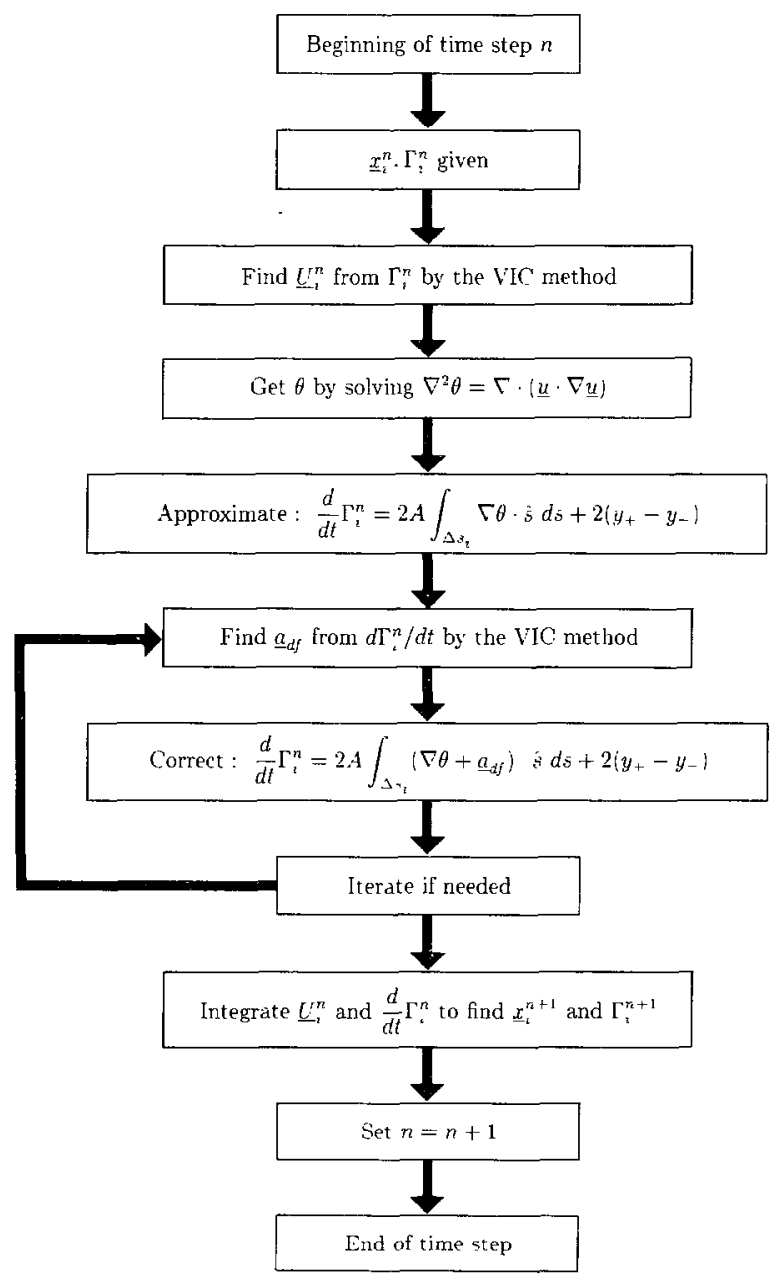

Fig. 2. Flowchart for the acceleration potential (AP) method. Here $\mathbf{a}_{d f}=\nabla \times(\phi \hat{k})$. 
The Iterative Time Step Methad

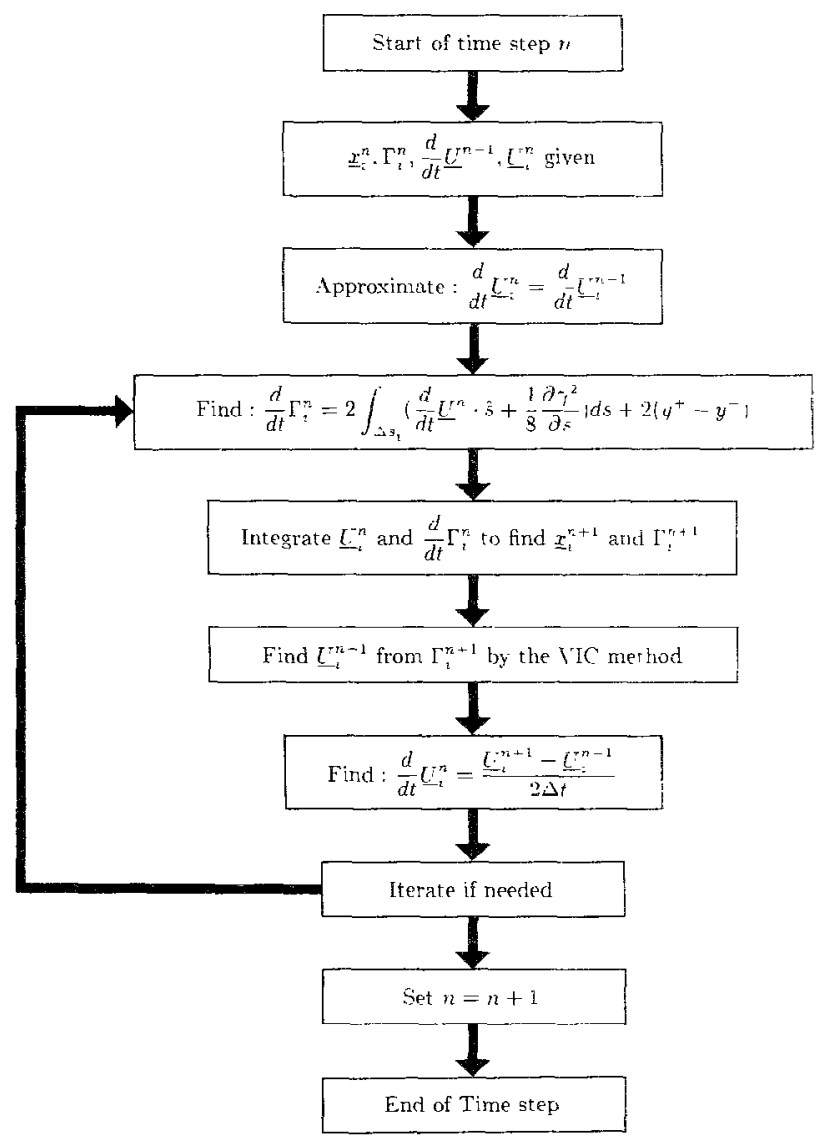

FIG. 3. Flowchart for iterative time step (ITS/ method.

To make the steps in each method somewhat clearer, a flowchart for each method is given in Figs. 2 and 3. The method based on the decomposition of the acceleration into an divergence-free part and curl-free part (the AP method) is shown in Fig. 2, and the method based on the iterated time stepping (the ITS method) is shown in Fig. $3 .^{2}$ For reasons of efficiency the actual code is slightly different from the flowcharts, but the flowcharts represent workable algorithms.

As in previous work $[55,56]$ it was necessary to occasionally redistribute the interface points. Since the cost of each run depends only weakly on the number of interface points, it was found convenient to use a relatively large number of points

2 The presentation of the methods by flowcharts was suggested to me by Professor N. J. Zabusky. 
and keep the redistributing algorithm rather primitive. In general the results were insensitive to the exact value of the redistributing parameters, as well as the number of interface points used. The main change to the redistributing process described in [55] was the addition of a point insertion routine, and that redistribution (and point addition) was now done automatically whenever the point distribution required (instead of every fixed number of time steps). For simulations employing direct summation methods, it is essential to keep the number of interface points as small as possible, and the redistribution must be done much more carefully, for discussions see, e.g., [60].

For small density differences the interface is unstable to Kelvin-Helmholtz shear instability and for fine grids the interface can develop small-scale rolled-up vortices from disturbances induced by the grid (see, e.g., Baker [5]). By smoothing the interface slightly these small disturbances can be dissipated. For calculations with the original (four-point) VIC method such smoothing was necessary for the first time steps in finely resolved runs. After a concentrated vortex had formed, the stretching of the interface was apparently sufficient to stabilize the grid generated disturbances and the smoothing was discontinued. For the coarser grids the wavenumber of the grid disturbances was sufficiently low (and therefore also their growth rate) so that when their growth was stopped by interface stretching, the disturbance amplitude was still small. However, it is interesting to note, that when no smoothing is applied on fine grids and many small vortices appear, these vortices usually merge into one big vortex and (except for the more complicated shape of the rolled-up region) the interface evolves similarly to when smoothing is applied and only one regular vortex appears. For simulations using the more isotropic VIC method, much less, and in many cases no, smoothing was needed.

\section{NumErical Results and DisCussion}

In this section the performance of the methods is discussed. The only "exact" solution available is the Baker, Meiron, and Orszag [6] and Menikoff and Zemach [35] solution for the single fluid case, so our main validation test for the two-fluid case is a study of the solution under grid refinement. If not stated otherwise the results presented were obtained using the modified VIC method.

The evolution of a single wave disturbance where the heavy fluid is three times as dense as the lighter fluid $(A=0.5)$ as calculated by the acceleration potential (AP) method is shown in Fig. 4. As expected, the heavy fluid falls into the lighter one and rolls up into two counter-rotating vortices with a slight downward velocity. The grid is 32 by 128 square meshes. In Fig. 5 the large amplitude configuration is compared for runs on different grids. Fig. 5a uses a 16 by 32 grid; $5 b$, a 32 by 64 grid (same resolution as in Fig. 4, but shorter computational box); and 5c, a 64 by 128 grid. The nondimensional time is 1.75 for all frames. The main differences between the figures is in the vortex. The vortices on the finest grid have a tightly wound spiral, where as on the coarsest grid there is very little roll-up. Outside the vortex 


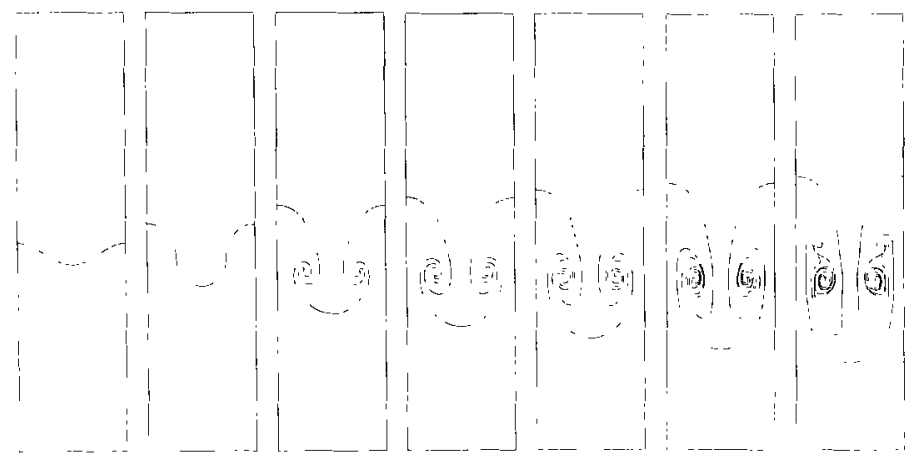

FIG. 4. The evolution of a single wavelength initial condition as calculated by the $\mathrm{AC}$ method using a modified VIC method. The heavy fluid is three times as dense as the lighter fluid, $A=0.5$. The grid is 32 by 128 meshes, and the initial amplitude was $10 \%$ of the wavelength. The iriterface is shown at times: $0.0,1.0,1.5,1.75,2.0 .2 .25$, and 2.5 .

the interfaces differ less, although the position of the vortices and the amplitude of the spike (we use the terminology of spike and bubble, although the distinction is not as clear as in the single fluid case) show a weak dependence on resolution (see Fig. 7). This nonuniform convergence, rapid outside the vortex, but slow (or possibly nonexisting) within the vortex, has recently been observed in studies of vortex sheet roll-up by the so-called vortex blob methods. For vortex sheets separating fluids of slightly different densities (Anderson [1]), and for vortex sheets in a constant density fluid (Krasny [27]), roll-up is a strong function of the size of the vortex blob employed, while outside the roll-up region the interface con-
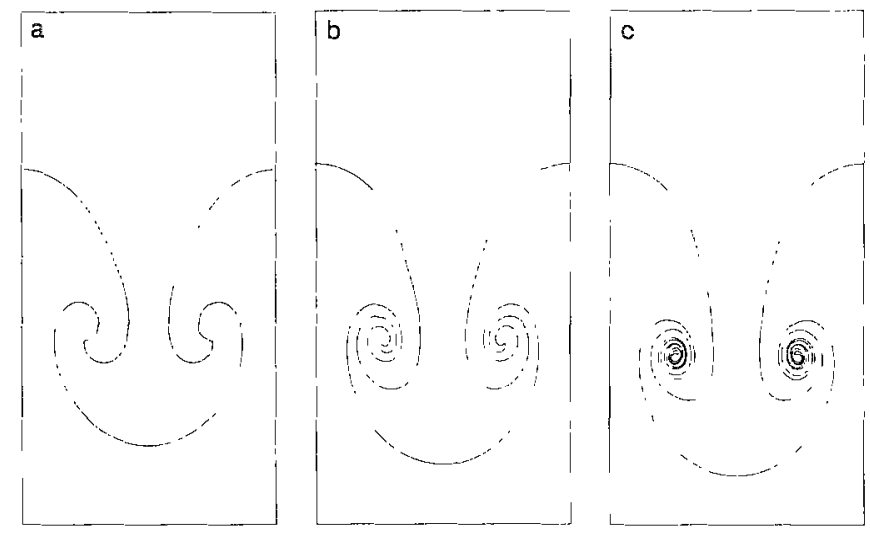

Fig. 5. The large amplitude stage at time 1.75 for the same densities as in Fig. 4 . but differen resolutions. The computational domain used here is shorter than in Fig. 4 : (a) 16 by 32 grid. (b) 32 by 64 grid (same resolution as in Fig. 4); and (c) 64 by 128 grid. As in Fig. 4 the AC method was used fo: these calculations. 
figuration is only slightly affected by the blob size. In the VIC method the interface vorticity is distributed to the nearest grid points, thus, in effect replacing the singular interface vortices by vortex blobs. The size of the resulting blob obviously depends on the mesh size, so it comes as no surprise that refining the grid has the same effect as reducing the blob size. The similarity between the VIC method and the vortex blob method for the situation considered by Krasny [27] is discussed by Tryggvason [54]. The lack of convergence is believed to be due to the formation of a singularity on the vortex sheet at the center of the roll-up. Numerical and asymptotic studies of this singularity formation for vortex sheets without any stratification include those of Moore [38]; Meiron, Baker, and Orszag [33]; and Krasny [26]. For real fluids the singularity is, of course, completely removed by viscosity, but it is presumably important to explore fully the nature of this phenomenon both for an improved understanding of the limitations of the vortex sheet model and for the possible generation of "weak" solutions of relevance to real physical flows.

In Fig. 6a the amplitude of the wave for the runs in Fig. 5 is plotted versus time. The bubble amplitude is indistinguishable between the two better resolved runs, indicating a fully converged solution. The downward motion of the spike is somewhat more resolution dependent, although the difference between the better resolved runs is less than between the coarser runs, indicating a convergence under grid refinement. Figure $6 \mathrm{~b}$ shows the velocity versus time. Again, the bubble velocity is fully converged in the better resolved runs, but the spike velocity less so. The waves on the velocity graph indicate that although grid effects have been reduced greatly by the use of a modified VIC method, they have not been completely eliminated. The bubble has reached a terminal velocity of about 0.265 in nondimensional units. The strong reduction in the spike velocity at the end of the run is due to the presence of the lower no-through flow boundaries. (The box boundaries are at 0.0 and 2.0 in Fig. 6a.) In order to estimate the asymptotic behavior of the spike, a longer box is required.

The resolution dependency of the downward motion of the spike increases with the density difference. The reason is that the term a $\cdot \hat{s}$, which is responsible for the advection of vortex sheet strength downward and hence any departure from symmetry, is calculated on the grid and not on the interface itself, as when the decomposition (Eq. (18)) is uscd. For large density differences this term is very significant, and it is essential that it is well resolved. The difficulty in doing so is the prime reason that this method is limited to low density differences. The poorly resolved $\mathbf{a} \cdot \hat{s}$ term will not advect the vorticity sufficiently rapidly toward the spike, hence the vortices will form earlier and closer to the center line than they should. In principle, very high resolution could be used, but when this was tried for the single fluid case $(A=1.0)$, numerical instabilities in the form of high wavenumber disturbances along the side of the spike appeared. These instabilities do not appear in the ITS method and must therefore be associated with the treatment of the $\mathbf{a} \cdot \hat{s}$ term. They are also not related to the Kelvin-Helmholtz instability sometimes encountered for small density differences, since this problem occurs only for large density differences 
where the interface is stable against Kelvin-Helmholtz instability. The exact cause of this instability has not been determined. High wavenumber instability for the single fluid case has been encountered in several simulations using direct summation methods, see, e.g., $[7,30,40]$.

In the second method described in Section 2. the iterative time step (ITS) method, the vorticity convection term a $\cdot \hat{s}$ is not resolved on the grid, but rather on the interface itself (using Eq. (18)). Therefore, it is to be expected that this method
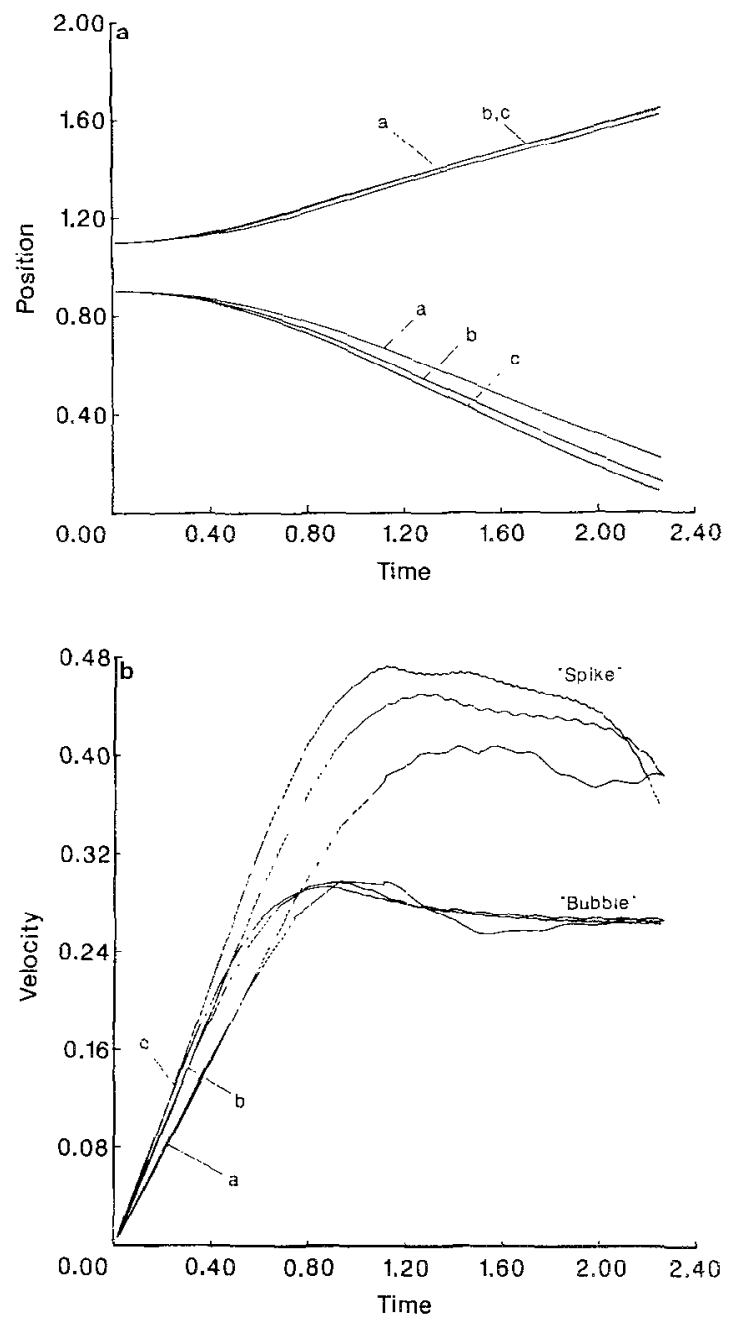

F1G. 6. (a) Amplitude versus time for the three runs in Fig. 5: (a) 16 by 32 grid: (b) 32 by 64 grid: and (c) 64 by 128 grid. (b) Velocity versus time for the three runs in Fig. $5:$ (a) 16 by 32 grid; (b) 32 by 64 grid; and (c) 64 by 128 grid. The spike velocity is reduced sharply at the end of the calculations due to the presence of the lower boundaries. 


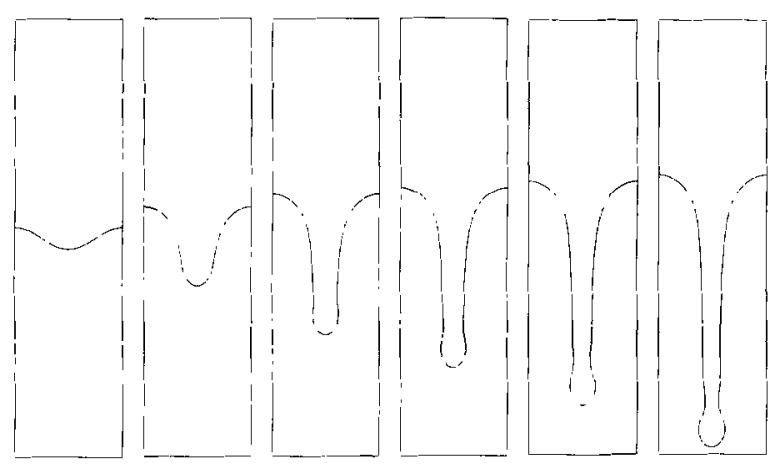

Fir. 7. The single fluid case $(A=1.0)$. Calculation is by the ITS method. The grid is 64 by 256 mesh and the initial amplitude was $10 \%$ of the wave length. The interface is shown at times: $0.0,1.0,1.5,1.75$, 2.0 , and 2.25 .

performs better when the density difference is large. This is indeed the case. Figure 7 shows the evolution of a single wave disturbance for $A=1.0$. The grid is 64 by 256 square meshes. The heavy fluid falls down forming a narrow "spike," while the lighter fluid rises upward in a big "bubble." These results compare rather well with those of Baker, Meiron, and Orszag [6] and Menikoff and Zemach [35], except that the tip of the spike should be sharper. The pendant drop at the tip of the spike is a numerical effect and is more pronounced for coarser resolutions. In Fig. 8 the large amplitude stage is compared for three different resolutions. The frames correspond to the same nondimensional times, $t=2.0$. Except for the spike, the interface configurations differ little, particularly for the two finer resolutions. Since the spike, gets thinner as it falls, it will always eventually become poorly resolved. Naturally, this happens first on the coarse grids. Insufficient resolution causes the spike to

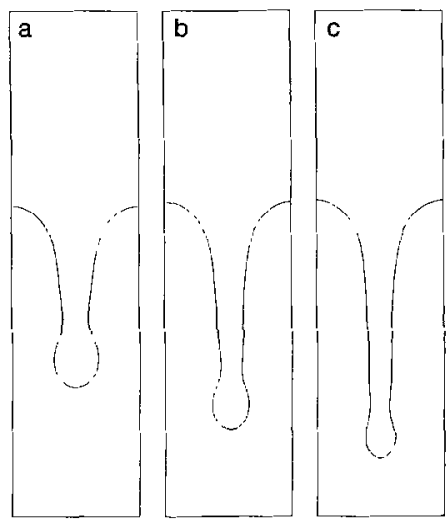

FIG. 8. The large amplitude stage for the single fluid case and three resolutions at time equal to 2.0 . Calculations are by the ITS method: (a) 16 by 64 grid; (b) 32 by 128 grid; and (c) 64 by 256 grid (same as in Fig. 7). 
terminate in a drop that falls considerably slower than a better resolved spike. In Fig. 9a the amplitudes of both the spike and the bubble versus time are compared for these three resolutions, as well as with the "exact" solution of Baker, Meiron, and Orszag [6]. The bubble amplitude is well reproduced, even on the coarse grid, but the spike appears to need considerable resolution in order to come close to the
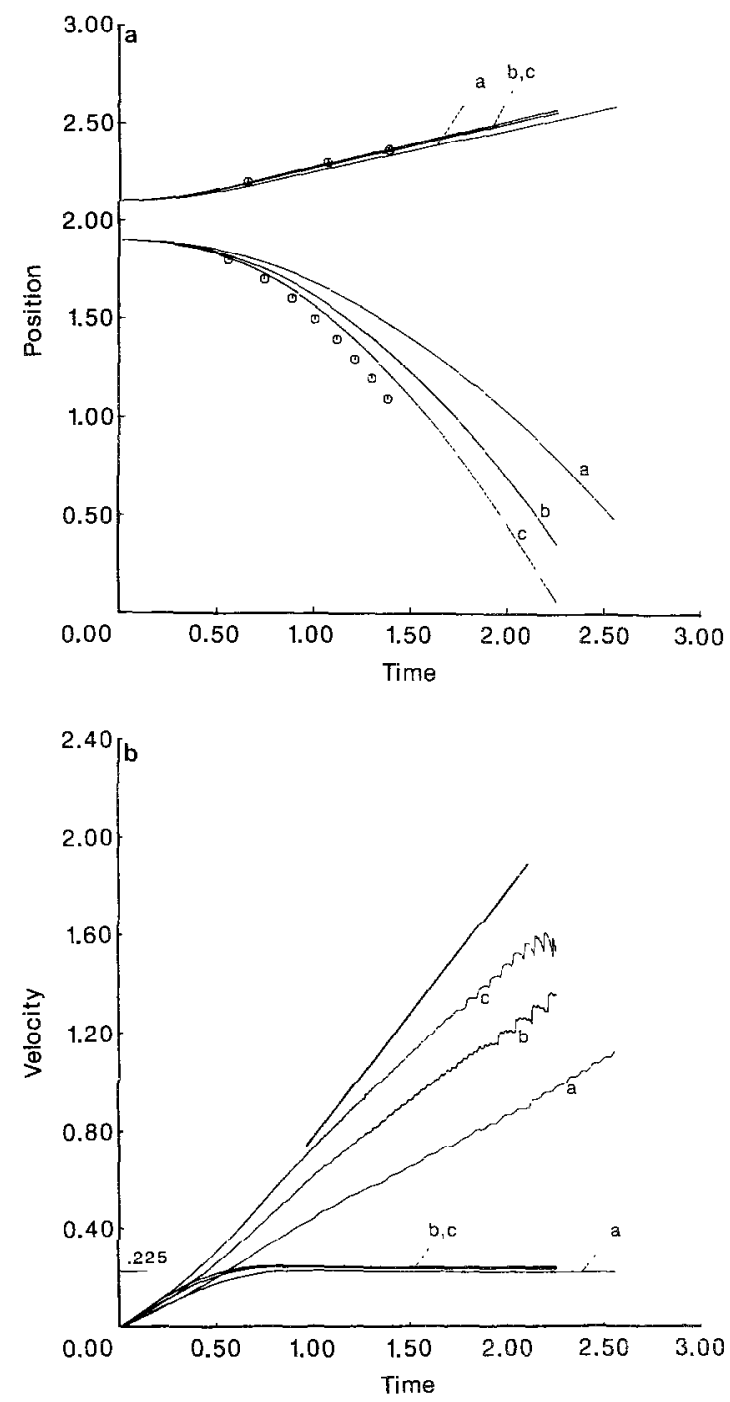

Fig. 9. (a) The amplitude versus time for the runs in Fig. 8, and the "exact solution" of Baker. Meiron, and Orszag [6]: (a) 16 by 64 , (b) 32 by 128 , and (c) 64 by 256 . Open circles: "exact solution." (b) The velocity versus time for the runs in Fig. 8 . The asymptotic velocity of the bubble predicted in [6] is marked on the vertical axis. The straight line is of slope 1 which is the asymptotic acceleration of the spike predicted in [6]. 
"exact" solution. However, the inaccurate representation of the spike does not seem to cause any significant distortion in the rest of the solution.

The reason the spike falls much slower on the coarse grid can be associated with the distribution of the interface vorticity onto the grid. The spike has a large vortex sheet strength of opposite sign on the sides and therefore may be considered as something like a dipole. When this vorticity of both signs is distributed on a coarse grid, cancellation occurs and the effective dipole strength, and hence the velocity, is reduced. The velocity of the bubble and the spike for the same runs is plotted in Fig. 9. The estimate for the terminal velocity of a bubble given by Baker, Meiron, and Orszag [6] is marked on the axis, and the inclined line represents the uniform acceleration of the spike predicted in the same reference. Although the spike falls short of reaching this acceleration in our simulations, it is noteworthy that apart from the actual numerical values, the behavior is as observed in $[6,35]$. The spike eventually falls with constant acceleration, but during the initial evolution the acceleration exceeds this asymptotic value. As for the lower density ratios the graphs contain some "wiggles" indicating that grid effects are not completely absent even though a modified VIC method is used.

Although the ITS method does relatively well for $A=1.0$, and the problem of cancellation of opposite strength vorticity does not occur for interfaces that roll up, a naive application to interfaces that roll up leads to failure. This is true even for low density differences where the advection terms should only be a small perturbation. This failure at amplitudes equal to one or two wavelengths is a common difficulty for other methods as well (see, e.g., the comment by Sharp [50]). This author has also found the same behavior when running the front tracking code of Glimm et al. [21]. Only on very coarse grids can these calculations be continued for longer time; more accurate calculations break down earlier.

The differences between the methods described here and their performances allow us to obtain some understanding of the problems encountered in the simulations of the Rayleigh-Taylor instability. For $A=0.0$ (the Boussinesq approximation) the a $\cdot \hat{s}$ term vanishes identically and the vorticity generation equation has a rather simple form. In many ways this problem is similar to the "ordinary" vortex sheet problem and remedies that work there should work here also. This is indeed the case, and both vortex blob methods (Anderson [1]) and the VIC method can be successfully applied. The cure, as discussed before, is to replace the singular vortex sheet at each time-step with a slightly smoother vorticity distribution. This prevents a singularity from appearing but makes the exact form of the rolled-up vortex dependent on the smoothness parameter (the mesh size for the VIC method). Outside the vortex, the interface is relatively insensitive to this parameter.

To treat the non-Boussinesq case $(A \neq 0)$ we have generated two methods, one that treats the advection terms as accurately as possible on the interface itself (the ITS method), and another that treats these terms in very much the same way as the vorticity and the velocities are treated in the VIC method, by smoothing the singular source terms onto a grid and thereby generating a grid acceleration field (the AP method). The reason for the difficulties in the ITS method appears to be 

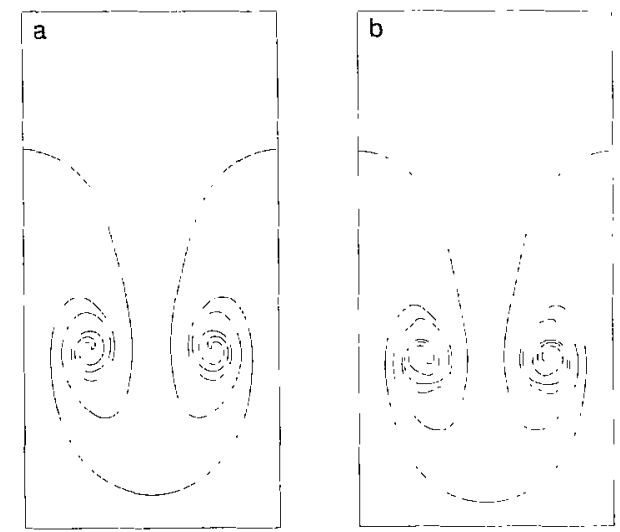

Fic. 10. Comparison of the large amplitude stage for both methods for moderate density differences $(A=0.5$. the heavier fluid three times denser than the light fluid). The nondimensional time is $2.0:$ (a) AP method and (b) ITS method. Explicit regularization of the vorticity advection term was used for the calculations in (b). The grid is 32 by 64 meshes in both cases.

that it is not sufficient to use a smooth vorticity field to find the velocity. It is also necessary to regularize the vorticity generation terms. This is accomplished in the AP method by calculating the $\mathbf{a} \cdot \hat{s}$ term on the grid. In the ITS method the $d \mathbf{U} / d t$ term is calculated from the already smooth velocity field, but $\partial \gamma^{2} / \partial s$ is not regularized at all. If $\gamma$ is singular, or nearly so, obviously $\partial \gamma^{2} / \partial s$ is likely to cause trouble. To account for this necessary, but missing, regularization in the ITS method we have explicitly regularized $\gamma$ at every time-step for use in the advection term in the vortex sheet equation (20). Many such regularizations are presumably possible; here we have simply used a running weighted average. Figure 10 shows

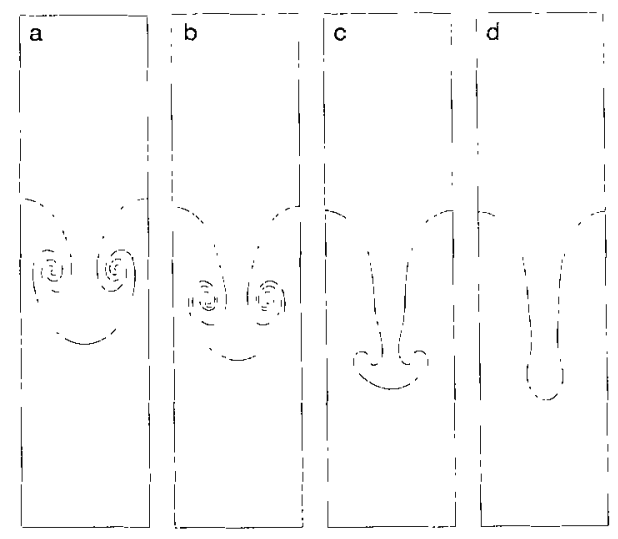

FIG. 11. Large amplitude stage for various density ratios, and the same initial condirions as calculated by the ITS method. The grids are 32 by 128 meshes and the nondimensional time is 1.75 in all cases: (a) $A=0.0$, (b) $A=0.5$, (c) $A=0.8$, and (d) $A=1.0$. Explicit regularization of the vorticity advection term was used for the calculations in (b), but not in (c) and (d). 

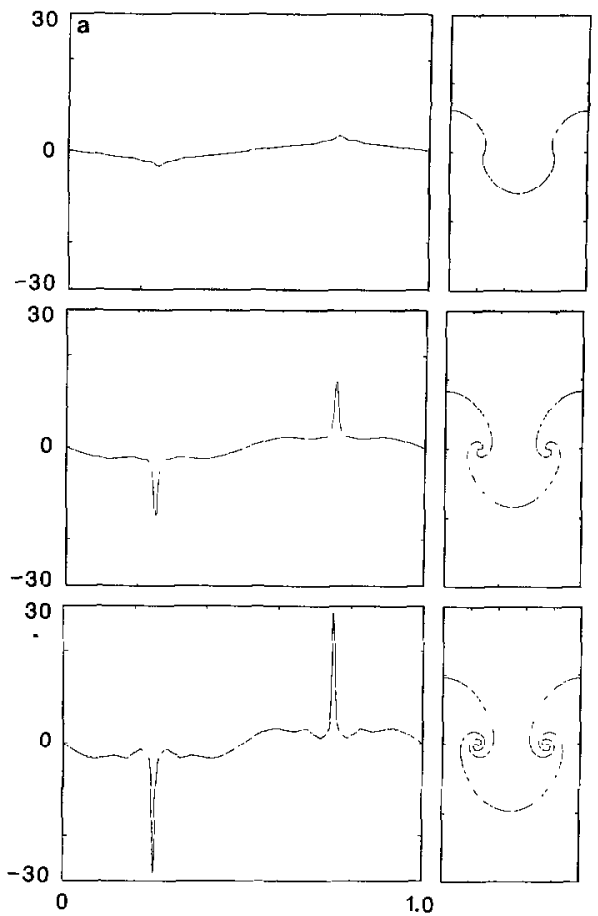
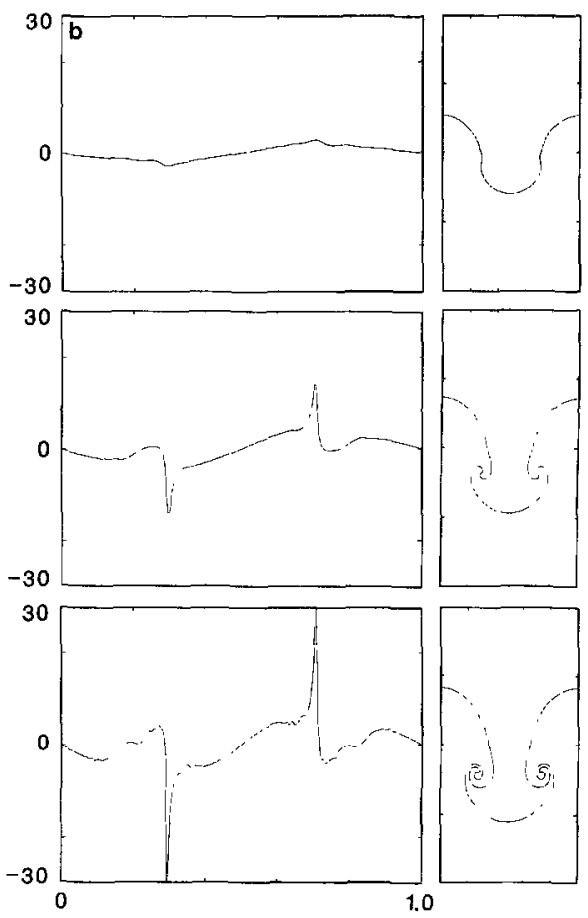

Fig. 12. The vortex sheet strength versus arc length: (a) $A=0$, (b) $A=0.6$, and (c) $A=1$. Notice that the scale for $\gamma$ in (a) and (b) is different from the scale in (c), and that the arc length is normalized by the total arc length. These calculations were done with the original four-point VIC method and the grid is 32 by 64 meshes in all cases. The "wiggles" in the curve in (c) are numerical, but did not grow in time.

the large amplitude configuration for $A=0.5$ on a 32 by 64 grid simulated by both methods, (a) AP method and (b) ITS method, using an explicit regularization for $\gamma$ before we calculate $\partial \gamma^{2} / \partial s$. Not only are the results quite similar, but the solution calculated by the ITS method is also rather insensitive to the exact form and value of the regularization used.

In Fig. 11 the difference in the configuration of the interface at large amplitudes for four different values of $A$ is shown, as calculated by the ITS method. The grid is 32 by 128 square meshes, and the frames correspond to approximately the same nondimensional time $(t=1.75)$. Figure 11 a is for $A=0.0 ;(\mathrm{b}), A=0.5$; (c), $A=0.8$; and (d), $A=1.0$. In 11(a) all the vorticity advection terms are identically zero. In 11 (b) smoothing (or regularization) is applied to $\gamma$ before $\partial \gamma^{2} / \partial s$ is calculated, but none is applied in (c) or (d). The $A=1$ case (d) can be calculated to much larger amplitudes without any such smoothing. Indeed it is important not to smooth, since the structure is quite sensitive to this particular term. The thick spike tip (pendant drop) is a numerical artifact due to the grid. Similar distortion is also observed if a blob is used in calculations working directly with the Biot-Savart integral. 


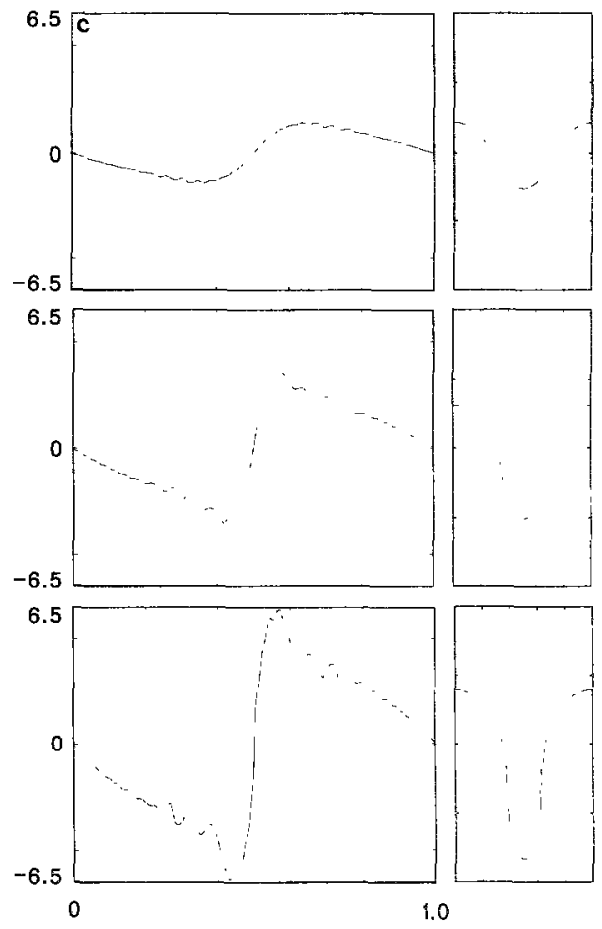

FigURE 12 (continued)

Detailed numerical tests indicate that the solution in (b) is relatively insensitive to the amount of smoothing applied to obtain a rcgular solution as shown here; less smoothing will produce essentially the same solution but with a somewhat more "irregular" vortex region. For $A=0.8$ case (c) this insensitivity was not observed, and no large range of the smoothing parameter was found for which the solution remained essentially unchanged. This may in part be due to the fact that the singularity forms much later in this case than for smaller density differences, and that smoothing may affect the evolution up to the singularity formation time too much. No particular difficulties are associated with simulations up to the time shown for $A=0.8$, but numerical difficulties show up shortly thereafter. Notice that even though the $c \gamma^{2} / \partial s$ term is not regularized, the inherent vortex blob regularization of the velocity field, due the grid, is still present. It is possible that a more selective smoothing, switched on at the time of the singularity formation and only applied where needed may produce better results. This possibility, as well as application of surface tension as a regularization mechanism, is under investigation. (Surface tension has been used before (e.g., Pullin [40]), with mixed success, it is possible that some additional dissipation is needed.)

To gain more insight into the behavior of the solution as the Attwood numbcr (the density ratio) is increased, the vortex sheet strength $\gamma$ is plotted versus arclength in Fig. 12 for $A=0.0$ (a), $A=0.6$ (b), and $A=1.0$ (c). (These particular 
simulations were done with the original VIC method.) Notice that the scale for $\gamma$ in (a) and (b) is different from the scale in (c) and that the arclength has been scaled by the total arclength in each case. The form of $\gamma(s)$ is very different for the two extreme density differences. For $A=0.0$ the vortex sheet strength exhibits a very strong spike lucated at the vortex center. The height of this spike is dependent of the resolution, thus suggesting the existence of a singularity. This process of vorticity intensification is made the basis of a model of weakly stratified RayleighTaylor flow in Aref and Tryggvason [4]. For $A=1.0$ the maximum (and minimum) vortex sheet strength is much smaller (notice the different scales), and the maxima and minima are close together. The change from one to the other takes place in an abrupt, almost shock-like, manner. The "wiggles" in the profile are numerical, but did not grow in time. For calculations using the modified VIC method these "wiggles" are smaller. It is clear that if the spike for $A=1.0$ does develop a singularity, then it must be of the form of a discontinuity in $\gamma(s)$, and hence, very different from the singularity for low values of $A$. For intermediate values of $A$, when roll-up still occurs, the spike in $\gamma^{\prime}(s)$ still forms, Fig. 12(b), but the distribution no longer has the same symmetries as for $A=0.0$. It therefore appears that for $A=1.0$, it is very important to account correctly for the advection of vorticity into the spike, and since no singularity appears to form, any desingularization may actually do damage since it usually involves some smoothing. The "shock" in $\gamma(s)$ for $A=1.0$ may also provide some clue as to why all methods encounter difficulties for nonsymmetric resolution of the spike, since any asymmetric resolution can generate large errors and instabilities. This numerical instability of unsymmetrically resolved spikes limits severely the calculations of general initial conditions in the single fluid case, even though no singularity appears to form. This problem at large amplitudes was also encountered by Baker, Meiron, and Orszag [6] and in simulations using the code of Glimm et al. [21]. It may also be mentioned that Sethian [48] has recently studied the propagation of a spike for a simplified set of model equations and encountered the same kind of difficulties. Only by resolving the spike exactly symmetrically could he follow the motion to large amplitude.

For finite density ratios it appears to be important to regularize the problem, not only the equation for the velocity but also the equation for the acceleration. This is accomplished in the AP method, but has to be inserted explicitly when the decomposition (18) is uscd. Wc are faced with a somcwhat contradictory task for high values of $A$ less than one. On the one hand we need to desingularize the problem to be able to simulate the roll-up, but on the other hand we have to advect the vorticity correctly downward, and to do so we do not want to smooth out the a $\cdot \hat{s}$ term. In the ITS method it is therefore presumably necessary to reduce the regularization as $A$ is increased. A systematic study of this has not been done, but a direct summation code extending vortex blob methods to stratified flows has recently been completed by the author. This type of grid-free method, although considerably more inefficient than the present methods, offers a "cleaner" environment (no grid disturbances) to study such delicate questions as singularity formations and how to apply proper regularizations. 


\section{CONCLUSION}

The problems encountered in simulating the Rayleigh-Taylor instability for inviscid, incompressible fluids have been discussed here. For low density differences (say $A \leqslant 0.6$ ) we have presented methods that are both robust and fast. We have simulated general initial conditions and have not encountered any problems for as long as we have run our programs. It is of course difficult to assess the accuracy at large amplitudes, and for large values of $A$, care must be taken that the resolution is sufficiently high. In Fig. 13 we present examples of runs with three different initial waves of random amplitude and phase. Figure 13(a) is for $A=0.0$ (the Boussincsq approximation) and $13(\mathrm{~b})$ is for $A=0.5$. The grid is 32 by 64 meshes. At first each wave grows relatively independent of the others to amplitudes roughly half the pertubation wavelength. Then the waves seem to interact strongly, and in the process, the largest wave develops into a dipole-like structure that may propagate out of the
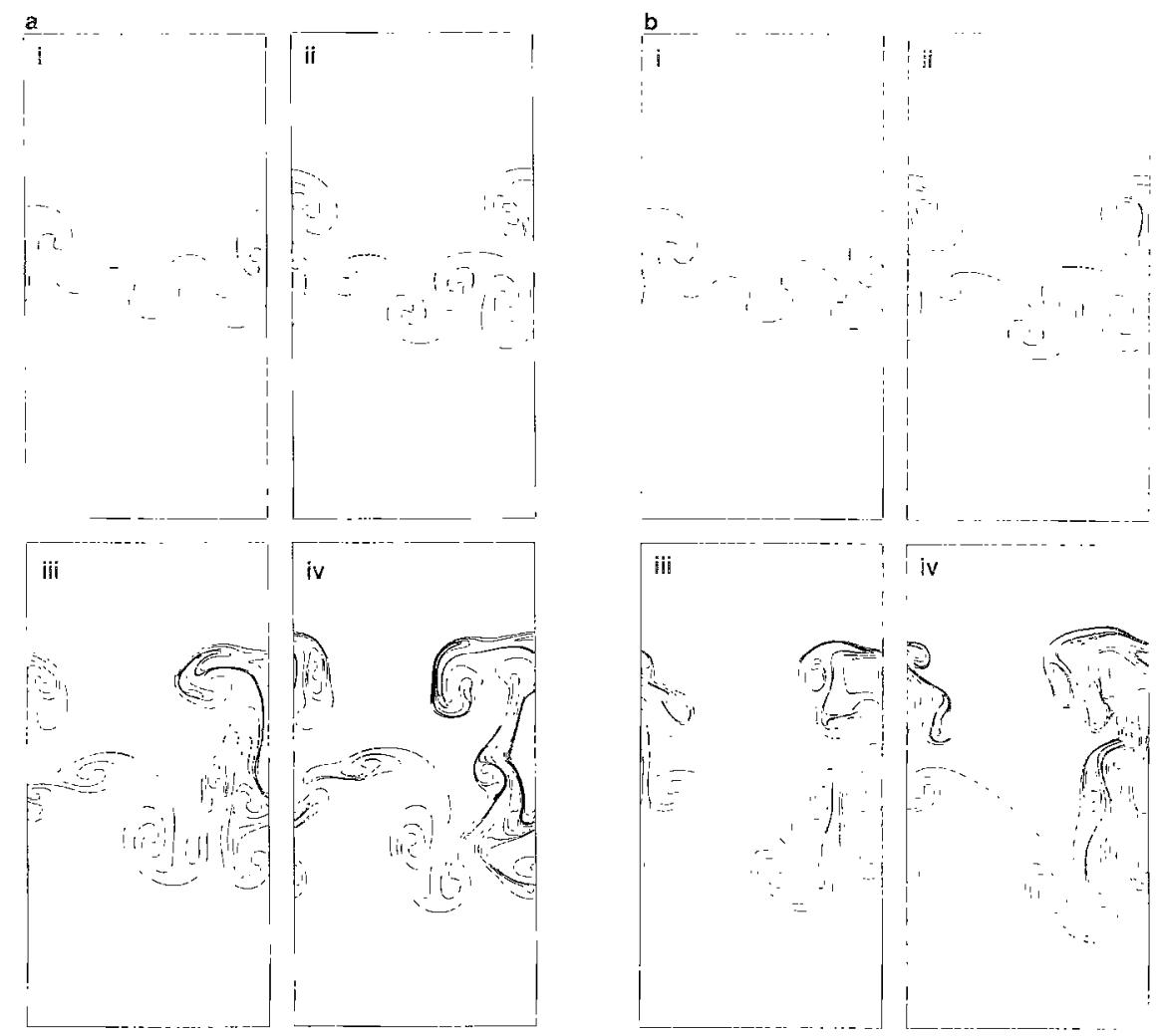

Fig. 13. The evolution of three waves of random amplitude and phase (on a 32 by 64 grid): (a) $A=0.0$ and (b) $A=0.5$. The initial conditions are $y(x)=0.125(0.7 \cos 2 \pi x+0.5 \sin 2 \pi x+$ $0.3 \cos 4 \pi x+0.5 \sin 4 \pi x+0.4 \cos 6 \pi x+0.3 \sin 6 \pi x$ ). The box is of unit width and two linits high. The times are: (i) 1.5 . (ii) 2.0 . (iii) 2.5 , and (iv) 2.75 . 

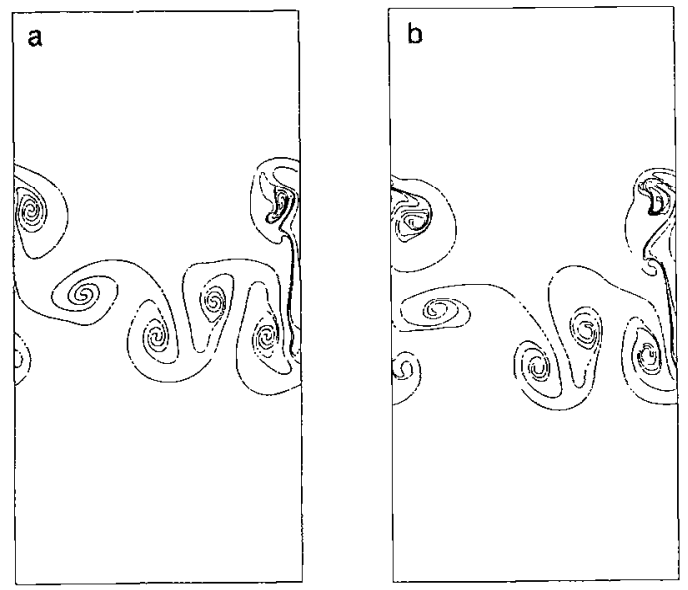

FIG. 14. The interface at time 2.0 for the same initial conditions as in Fig. 13, but on a 64 by 128 grid: (a) $A=0.0$ and (b) $A=0.5$.

main mixing zone. Notice that the interaction of the large amplitude vortices leads to an increase in "mean length scale" from three initial waves to one agglomeration of several vortices. Obviously, it is necessary to perform larger simulations to determine to what extent the mixing is affected by the formation of dipole-like structures, what their fate is at large amplitude, and how the large amplitude interactions change the length scales in the mixed zone. Larger simulations should present no problems since these runs require only modest amounts of computer time. The prime limiting factor at the moment is the inconvenience associated with transferring large amounts of data between sites. The last frame in Figs. 13(a) and (b) (and Fig. 14) contains several thousand points. Since these simulations use a rather coarse grid (corresponding to large vortex blobs) relatively little roll-up has taken place in the core of the vortices. Figure 14 shows the same initial conditions at $t=2.0$ calculated on twice as fine a grid, where (a) is for $A=0.0$ and (b) is for $A=0.5$. Although the interface configuration is not exactly identical to the corresponding frame in Fig. 13, close inspection reveals that the difference is mostly due to more roll-up in the vortices in Fig. 14. The instability on the coarse grid also evolves slightly slower than on the finer grid. The properties of the mixing due to a Rayleigh-Taylor instability will be discussed in dctail in futurc publications.

\section{ACKNOWLEDGMENTS}

This work was initiated while I was at Brown University working with Professor Hassan Aref. His suggestions are too numerous to list. At Brown this work was partially supported by NSF Grant MEA 81-16910. Further work was done at the Courant Institute of Mathematical Sciences with partial support by NSF Grant DMS 8312229 . Most recently, this work has been supported by a Rackham faculty grant 
from the Unversity of Michigan, and by the Naval Research Laboratory (Contract N00014-83-C-2006). I wish to acknowledge constructive interactions with Professors J. Glimm and N. I. 7abusky, and Drs. D. H. Sharp and R. Menikoff. The computations were done on the computers at NCAR. at the Courant Institute, and at SDSC. NCAR and SDSC are sponsored by the NSF.

\section{REFERENCES}

1. C. R. Anderson. J. Comput. Phys. 61, 417 (1985).

2. C. R. Anderson, J. Comput. Phys. 62, 111 (1986).

3. H. Aref and E. D. Siggia, J. Fluid Mech. 100, 705 (1980).

4. H. AREF AND G. TRYGGVASON, in preparation.

5. G. R. Baker, J. Comput. Phys. 31, 76 (1979).

6. G. R. Baker, D. I. Meiron, and S. A. Orszag, Phys. Fluids 23, 1485 (1980).

7. G. R. Baker, D. I. Meiron, and S. A. Orszag, J. Fluid Mech. 123, 477 (1983).

8. R. Bellman and R. H. Pennington, Quart. J. Appl. Math. 12, 151 (1954).

9. P. A. Bernhardt and J. U. Brackbill, J. Comput. Phys. 53. 382 (1984).

10. G. Birkhoff, Los Alamos Sci. Lab. Rep. LA-1862; appendices in Re. LA-1927. 1954 (unpublished;:

11. G. Birkhoff and D. Carter, J. Math. Mech. 6, 769 (1957).

12. O. Buneman, J. Comput. Phy's. 11, 250 (1973).

13. S. Chandrasekhar, Proc. Cambridge Philos. Soc. 51. 162 (1955).

14. A. J. Chorin and P. S. Bernard, J. Comput. Phys. 13, 423 (1973).

15. J. P. Christiansen, J. Comput. Phys. 13, 363 (1973).

16. B. J. DaLy, Phys. Fluids 10, 297 (1967).

17. M. H. Emery, J. H. Gardner, J. P. Boris, And A. L. Cooper, Phys. Fluids 27, 1338 11984)

18. H. W. Emmons, C. T. Chang, and B. C. Watson, J. Fluid Mech 7. $177(1960)$.

19. P. R. Garabedian, Proc. R. Soc. London A 241, 423 (1957).

20. P. R. Garabedian. Commun. Pure Appl. Math. 38, 609 (1985).

21. J. Glimm, O. McBryan. R. Menikoff, and D. H. Sharp, SIAM J. Sci. Slat. Comput. 7. 230 11987).

22. F. H. Harlow and J. E. Welch, Phys. Fluids 8, 2182 (1965).

23. J. J. L. Higdon and C. Pozrikidis, J. Fluid Mech. 150, 203 (1985।.

24. C. W. Hirt and B. D. Nichols, J. Comput. Phys. 39, 201 (1981).

25. R. W. Hockney, S. P. Goel and J. W. Eastwood, J. Cempul. Phys. 14, 148 (1974).

26. R. KRASNY. J. Fluid Wech. 167, 65 (1986).

27. R. KRasNy. J. Comput. Phys. 65, 292 (1986).

28. A. Leonard, J. Comput. Phys. 37, 289 (1980).

29. D. J. LEwIs, Proc. R. Soc. London A 202, 81 (1950).

30. M. S. Longuet-Higgins and E. D. Cokelet, Proc. R. Soc. London $A$ 364, 1 (1976).

31. H. J. Lugt. Vortex Flow in Nature and Technology. (Wiley, New York, 1983), p. 157.

32. A. MAYO, SlAM J. Numer. Anal. 21, 285 (1984)

33. D. I. Meiron, G. R. Baker and S. A. OrszaG, J. Fluid Mech. 114, 283 (1982).

34. J. C. S. Mteng and J. A. L. Thomson, J. Fluid Mech. 84, 433 (1978).

35. R. C. Menikoff and C. Zemach, J. Comput. Phys. 51, 28 (1983).

36 M. Mitchner and R. K. M. Landshoff, Phy's. Fluids 7, 862 (1964).

37. D. W. MoOre, J. Fluid Mech. 63, 225 (1974).

38. D. W. Moore, Proc. R. Soc. London A 365, 105 (1979).

39. C. S. PESk.IN, J. Comput. Phys. 25, 220 (1977).

40. D. I. Pullin. J. Fluid Mech. 119, 507 (1982).

41. H. Ramberg, Geophys. J. 14, 307 (1967).

42. K. I. READ, Physica D 12, 45 (1984).

43. Lord Ray leigh, Scientific Papers, Vol. ii, p. 200 (Cambridge, 1900). 
44. P. J. RoACHE, Computational Fluid Dynamics, revised ed. (Hermosa, Albuquerque, NM, 1982), p. 184.

45. J. W. Rottman and D. B. OlfF, AIAA J. 15, 1214 (1977).

46. P. G. Saffman and G. R. Baker, Annu. Rev. Fluid Mech. 11, 95 (1979).

47. J. Serrin, in Handbuch der Physik, Band VIII/1, edited by S. Flugge (Springer, New York/Berlin, 1959), p. 125.

48. J. Sethian, Commun. Math. Phys. 101, 4 (1985).

49. L. F. Shampine AND M. K. Gordon, Computer Solution of Ordinary Differential Equations (Freeman, San Francisco, 1975).

50. D. H. Sharp, Physica D 12, 3 (1984).

51. G. I. TaYlor, Proc. R. Soc. London A 201. 192 (1950).

52. G. Tryggvason, in Woods Hole Oceanographic Institulion Tech. Rep. WHO I-83-41, 1983, p. 207 (unpublished).

53. G. TryggVason. thesis, Brown University, 1985 (unpublished).

54. G. Truggvason, Simulation of vortex sheet roll-up by vortex methods, submitted for publication, 1987.

55. G. Tryggvason and H. Aref, J. Fluid Mech. 136, 1 (1983).

56. G. Triggvason and H. AREF, J. Fluid Mech. 154, 284 (1985).

57. C. P. Verdon, R. L. MCCrory, R. L. Morse, G. R. Baker, D. I. Meiron, and S. A. Orszag, Phys. Fluids 25, 1653 (1982).

58. D. L. Youngs, in Numerical Methods for Fluid Dynamics, edited by K. W. Morton and M. J. Baines (Academic Press. New York/London, 1982).

59. D. L. YOUnGS, Physica D 12, 32 (1984).

60. N. J. Zabusky and E. A. Overman, II, J. Comput. Phys. 52, 351 (1983).

61. R. G. ZALOSH, AIAA J. 14, 1517 (1976).

62. S. J. Zaroodny and M. D. Greenberg, J. Comput. Phys. 11, 440 (1973). 